\title{
Порядок Шарковского
}

\section{и оценки числа периодических траекторий данного периода отображений отрезка}

\author{
О. А. Иванов \\ Санкт-Петербургский государственный университет, \\ Российская Федерация, 199034, Санкт-Петербург, Университетская наб., 7-9
}

Для цитирования: Иванов О.А. Порядок Шарковского и оценки числа периодических траекторий данного периода отображений отрезка // Вестник Санкт-Петербургского университета. Математика. Механика. Астрономия. 2019. Т. 6 (64). Вып. 3. С. 422-429. https://doi.org/10.21638/11701/spbu01.2019.307

В 1964 году А. Н. Шарковский опубликовал статью, в которой было введено отношение порядка на множестве натуральных чисел, обладающее тем свойством, что если у отображения отрезка в себя имеется периодическая траектория некоторого периода, то у этого отображения есть периодические траектории любого большего периода. Наименьшим числом относительно этого отношения порядка является число 3. Таким образом, если у отображения отрезка в себя есть траектория периода 3 , то у него есть траектории любых периодов. В 1975 году последний результат был переоткрыт Ли и Йорком, опубликовавшим статью «Period three implies chaos». Их работа привела к международному признанию результата, полученного Шарковским. С тех пор было опубликовано огромное число работ, связанных с изучением свойств отображений отрезка. А в 1994 году даже была проведена конференция «Thirty years after Sharkovskii's theorem: New perspectives». Одно из направлений исследований было связано с оценкой числа периодических траекторий, которое должно иметь отображение, удовлетворяющее условиям теоремы Шарковского. В 1985 году Бау-Сен Ду опубликовал работу, в которой была получена точная оценка снизу для числа периодических траекторий заданного периода. В настоящей статье приводится новое, существенно более короткое и естественное доказательство этого результата.

Ключевые слова: порядок Шарковского, отображения отрезка, периодическая траектория, пути на ориентированном графе.

1. Введение. В своей знаменитой работе [1] А. Н. Шарковский ввел отношение линейного порядка $m \triangleright k$ на множестве натуральных чисел, обладающее следующим свойством: если у отображения отрезка в себя есть точка периода $m$, то у него есть точки периода $k$. При этом им было показано, что для каждого натурального числа $m$ существует отображение, имеющее траекторию периода $m$ и не имеющее траекторий бо́льших (по отношению к введенному порядку) периодов. В частности, если $m=2 n+1$, то существует отображение с единственной периодической траекторией периода $m$, у которого нет траекторий меньших нечетных периодов (за естественным исключением единственной неподвижной точки этого отображения). Естественным образом возникает вопрос о числе траекторий периодов $k \triangleleft m$, которые могут иметь подобное отображение. В работе [2] была получена точная оценка снизу на число таких траекторий. Одним из результатов статьи [3] была точная

(c) Санкт-Петербургский государственный университет, 2019 
оценка снизу на число периодических траекторий отображения, имеющего траекторию периода 3 (во время подготовки к печати этой статьи автор еще не был знаком с работой [2]).

Собственно говоря, в [2] исследовалось число периодических траекторий конкретного кусочно-линейного отображения $f_{n}$ отрезка $[1 ; 2 n+1]$ в себя (заметим, что оно было введено еще в статье [1], см. также [4, 5]), которое не имеет периодических траекторий нечетных периодов, меньших $2 n+1$, а числа $\{1,2, \ldots, 2 n+1\}$ образуют траекторию периода $2 n+1$. В силу теоремы 2 работы [4], всякое отображение $f$ этого отрезка, у которого нет периодических траекторий нечетных периодов, меньших $2 n+1$, имеет по крайней мере столько же траекторий заданного периода, сколько их есть у отображения $f_{n}$. Поэтому число периодических траекторий отображения $f_{n}$ является оценкой снизу для числа траекторий произвольного непрерывного отображения с указанным свойством.

Основным результатом данной работы является новое, существенно более короткое и естественное доказательство основной теоремы работы [2]. Его основная идея состоит в использовании матрицы смежности для подсчета числа замкнутых путей заданной длины на некотором ориентированном графе с $2 n$ вершинами. Из этого доказательства также становится ясно, по какой причине при $n=1$ в формулах для числа траекторий заданного периода возникают числа Люка $L_{k}$.

2. Вспомогательное алгебраическое утверждение. Рассмотрим квадратные матрицы размером $n \times n$

$$
B=\left(\begin{array}{ccccc}
1 & 1 & \ldots & 1 & 1 \\
0 & 0 & \ldots & 0 & 1 \\
0 & 0 & \ldots & 1 & 0 \\
\vdots & \vdots & . & \vdots & \vdots \\
0 & 1 & \ldots & 0 & 0
\end{array}\right), C=\left(\begin{array}{cccc}
0 & \ldots & 0 & 1 \\
0 & \ldots & 1 & 0 \\
\vdots & . \cdot & \vdots & \vdots \\
1 & \ldots & 0 & 0
\end{array}\right) \text { и } D=\left(\begin{array}{cccc}
1 & 0 & \ldots & 0 \\
0 & 0 & \ldots & 0 \\
\vdots & \vdots & \vdots & \vdots \\
0 & 0 & \ldots & 0
\end{array}\right)
$$

и положим

$$
A=\left(\begin{array}{cc}
\mathbf{0} & B \\
C & D
\end{array}\right)
$$

где через 0 обозначена нулевая матрица. Пусть $\ell_{k}=\operatorname{Tr}\left(A^{k}\right)-$ это след $k$-й степени матрицы $A$, где $k=1,2, \ldots$

Лемма 1. Последовательность $\ell_{k}$ удовлетворяет рекуррентному соотношению

$$
x_{k+2 n}=x_{k+2 n-1}+\sum_{i=0}^{2 n-2}(-1)^{i} x_{k+i},
$$

при этом $\ell_{2 i-1}=1$ u $\ell_{2 i}=2^{i+1}-1$ nри $i=1,2, \ldots, n$.

ДокАЗАтЕльство. Непосредственно проверяется, что следующий многочлен является характеристическим многочленом матрицы $A$ :

$$
\varphi_{2 n}(t)=t^{2 n}-t^{2 n-1}+\sum_{i=0}^{2 n-2}(-1)^{i+1} t^{i}
$$


В силу теоремы Гамильтона - Кэли имеет место матричное соотношение $\varphi_{2 n}(A)=\mathbf{0}$,

$$
A^{2 n}-A^{2 n-1}+\sum_{i=0}^{2 n-2}(-1)^{i+1} A^{i}=\mathbf{0},
$$

поэтому

$$
A^{2 n+k}=A^{2 n+k-1}+\sum_{i=0}^{2 n-2}(-1)^{i} A^{k+i}
$$

следовательно,

$$
\operatorname{Tr}\left(A^{2 n+k}\right)=\operatorname{Tr}\left(A^{2 n+k-1}\right)+\sum_{i=0}^{2 n-2}(-1)^{i} \operatorname{Tr}\left(A^{k+i}\right)
$$

Таким образом, $\ell_{k+2 n}=\ell_{k+2 n-1}+\sum_{i=0}^{2 n-2}(-1)^{i} \ell_{k+i}$, т. е. рассматриваемая последовательность действительно удовлетворяет соотношению (2). Для поиска первых членов этой последовательности рассмотрим многочлен $\chi(t)=(t+1) \varphi_{2 n}(t)=$ $t^{2 n+1}-2 t^{2 n-1}-1$, корнями которого являются числа $\lambda_{1}, \lambda_{2}, \ldots, \lambda_{2 n}, \lambda_{2 n+1}=-1$, где $\lambda_{i}, i=1,2, \ldots, 2 n,-$ корни многочлена $\varphi_{2 n}(t)-$ характеристического многочлена матрицы $A$.

Рассмотрим степенные суммы $s_{k}=\sum_{i=1}^{2 n+1} \lambda_{i}^{k}$. Поскольку $\ell_{k}=\operatorname{Tr}\left(A^{k}\right)=$ $\sum_{i=1}^{2 n} \lambda_{i}^{k}$, то $s_{k}=\ell_{k}+(-1)^{k}$. Ясно, что $s_{1}=0$, а $s_{2}=4$. Из формул Ньютона, выражающих степенные суммы через элементарные симметрические многочлены (см., к примеру, [6, с. 266]), следует, что $s_{k+2}=2 s_{k}$ при всех $k=2,3, \ldots, 2 n-2$. Следовательно, $s_{1}=s_{3}=\ldots=s_{2 n-1}=0$, откуда и следует, что $\ell_{1}=\ell_{3}=\ldots=\ell_{2 n-1}=1$. Далее, $s_{2 k}=2 s_{2 k-2}=4 s_{2 k-4}=\ldots=2^{k-1} s_{2}=2^{k+1}$, поэтому $\ell_{2 k}=2^{k+1}-1$ при всех $k=1,2, \ldots, n$.

\section{Следствия.}

1. Справедливо соотношение $\ell_{k+2 n+1}=2 \ell_{k+2 n-1}+\ell_{k}$, при этом $\ell_{2 n+1}=2 n+2$.

2. Равенство $\ell_{2 i}=2^{i+1}-1$ имеет место при всех $i=1,2, \ldots, 2 n$.

Замечание. Если $n=1$, то $A=\left(\begin{array}{ll}0 & 1 \\ 1 & 1\end{array}\right)$, значит, $A^{k}=\left(\begin{array}{cc}F_{k-1} & F_{k} \\ F_{k} & F_{k+1}\end{array}\right)$, таким образом, $\ell_{k}=\operatorname{Tr}\left(A^{k}\right)=F_{k-1}+F_{k+1}=L_{k}$ (здесь $F_{i}-$ числа Фибоначчи).

3. Формулировка основного результата. Пусть отображение $f:[1 ; 2 n+1] \rightarrow$ $[1 ; 2 n+1]$ в натуральных точках этого отрезка определено посредством равенств

$$
\begin{gathered}
f(1)=n+1, \quad f(k)=2 n+3-k \text { при } k=2,3, \ldots, n+1, \\
f(k)=2 n+2-k \text { при } k=n+2, n+3 \ldots, 2 n+1,
\end{gathered}
$$

и является линейным на каждом из отрезков $J_{k}=[k ; k+1], k=1,2, \ldots, 2 n$. На рис. 1 изображен график отображения $f:[1 ; 7] \rightarrow[1 ; 7]$ для $n=3$.

Нетрудно видеть, что числа $\{1,2, \ldots, 2 n+1\}$ образуют траекторию периода $2 n+1$ отображения $f$. Действительно, имеем

$$
1 \stackrel{f}{\mapsto} n+1 \stackrel{f}{\mapsto} n+2 \stackrel{f}{\mapsto} n \stackrel{f}{\mapsto} n+3 \stackrel{f}{\mapsto} n-1 \stackrel{f}{\mapsto} \ldots \stackrel{f}{\mapsto} 2 \stackrel{f}{\mapsto} 2 n+1 \stackrel{f}{\mapsto} 1 .
$$




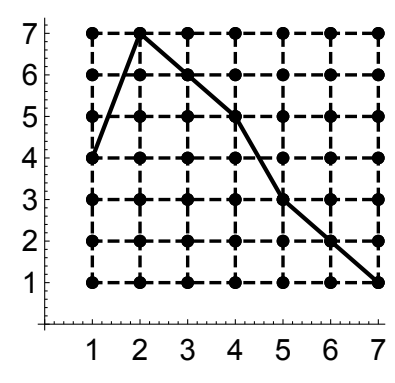

Рuc. 1. График отображения $f$ в случае $n=3$.

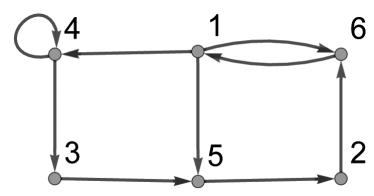

Puc. 2. Граф $\mathcal{B}_{6}$.

Основной результат работы [2] - это формула для числа траекторий периода $k$ введенного отображения $f$. Оказывается, что эту формулу можно записать в очень простом виде.

Теорема 1. Число траекторий периода $k$ отображения $f:[1 ; 2 n+1] \rightarrow[1 ; 2 n+$ 1] определяется по формуле

$$
\operatorname{traj}_{k}=\frac{1}{k} \sum_{d \mid k} \mu(d) \ell_{k / d}
$$

где $\ell_{i}$ - это последовательность следов степеней $A^{i}$ матрицы $A$ (см. лемму 1 и следствие $1 \kappa$ ней), н есть функиия Мёбиуса, а суммирование производится по всем делителям числа $k$.

4. Построение вспомогательного графа. Доказательство теоремы 1 основано на свойствах некоторого ориентированного графа $\mathcal{B}_{2 n}$. Его вершинами являются точки $\{1,2, \ldots, 2 n\}$. В этом графе имеется ребро $u \rightarrow v$, если $f\left(J_{u}\right) \supset J_{v}$. Будем задавать этот граф посредством его матрицы смежности $A=\left(a_{i j}\right)_{i, j=1,2, \ldots, 2 n}$, считая, что $a_{i j}=1$, если в графе имеется ребро $i \rightarrow j$ (остальные элементы этой матрицы полагаем равными нулю).

Для случая $n=3$ отображения $f:[1 ; 7] \rightarrow[1 ; 7]$ мы получим граф $\mathcal{B}_{6}$ (см. рис. 2). Его матрицей смежности является матрица

$$
A=\left(\begin{array}{llllll}
0 & 0 & 0 & 1 & 1 & 1 \\
0 & 0 & 0 & 0 & 0 & 1 \\
0 & 0 & 0 & 0 & 1 & 0 \\
0 & 0 & 1 & 1 & 0 & 0 \\
0 & 1 & 0 & 0 & 0 & 0 \\
1 & 0 & 0 & 0 & 0 & 0
\end{array}\right) .
$$

Следующее утверждение очевидным образом следует из определений отображения $f$ и графа $\mathcal{B}_{2 n}$.

Лемма 2. Матрица А, заданная равенством (1), является матрицей смежности графа $\mathcal{B}_{2 n}$. 
Под замкнутым путем на ориентированном графе мы будем понимать путь, в котором конечная точка совпадает с начальной. Следующее утверждение хорошо известно.

Лемма 3. Число замкнутых путей длины $k$ на ориентированном графе совпадает со следом $k$-й степени матрищы смежности этого графа.

Следующее утверждение является непосредственным следствием лемм 1, 2 и 3.

Лемма 4. Число замкнутых путей длины $k$ на графе $\mathcal{B}_{2 n}$ равно значению члена $\ell_{k}$ последовательности, определенной соотношением (2).

В дальнейшем нам потребуется следующее очевидное свойство графа $\mathcal{B}_{2 n}$ : всякий замкнутый путь на графе $\mathcal{B}_{2 n}$, за исключением постоянного пути $n+1 \rightarrow n+1 \rightarrow$ ..., проходит через вершину 1.

5. Доказательство теоремы 1. Как обычно, для произвольной точки $x_{1}$ через $x_{k}, k \geq 2$, обозначим точки ее траектории под действием отображения $f, x_{k+1}=$ $f\left(x_{k}\right)$. Следующее утверждение вполне стандартно для работ, посвященных данной тематике (см. $[1,3,7,8])$, и его доказательство приводится здесь только для полноты изложения.

Лемма 5. Для каждого замкнутого пути $u_{1} \rightarrow u_{2} \rightarrow \ldots \rightarrow u_{k} \rightarrow u_{1}$ на графе $\mathcal{B}_{2 n}$ существует единственная точка $x_{1} \in J_{u_{1}}$, порождаюшая траекторию отображения $f$, такую, что $x_{i} \in J_{u_{i}}$ при $i=1,2, \ldots, k$ u $x_{m+k}=x_{m}$ при всех натуральных $\mathrm{m}$.

Пусть $x_{1}, x_{2}, \ldots, x_{k}$ - точки искомой траектории. Поскольку $x_{k} \in J_{u_{k}}$, a $f\left(x_{k}\right)=$ $x_{1} \in J_{u_{1}}$, то $x_{k} \in J_{u_{k}} \cap f^{-1}\left(J_{u_{1}}\right)$. Положим $J^{(k)}=J_{u_{k}} \cap f^{-1}\left(J_{u_{1}}\right)$. Поскольку $x_{k-1} \in$ $J_{u_{k-1}}$ и $f\left(x_{k-1}\right)=x_{k} \in J^{(k)}$, то $x_{k-1} \in J^{(k-1)}=J_{u_{k-1}} \cap f^{-1}\left(J^{(k)}\right)$. Аналогичным образом строим отрезки $J^{(m)}=J_{u_{m}} \cap f^{-1}\left(J^{(m+1)}\right), m=k-2, k-3, \ldots, 1$.

Таким образом, если искомая точка существует, то она должна лежать в отрезке $J^{(1)}$. По построению $f^{k}\left(J^{(1)}\right)=J_{u_{1}} \supset J^{(1)}$, Значит, существует точка $x_{1} \in J^{(1)}$, такая что $f^{k}\left(x_{1}\right)=x_{1}$. Для доказательства единственности этой точки осталось заметить, что, как было замечено, всякий непостоянный замкнутый путь проходит через вершину 1. Поэтому, если $x \in J^{(1)}$, то $f^{k}(x)=a x+b$, где $|a|>1$, а потому уравнение $a x+b=x$ имеет не более одного решения.

Перейдем непосредственно к доказательству теоремы 1 . Обозначим через $p_{k}$ количество точек, лежащих на траекториях периода $k$. Ясно, что каждой такой точке можно сопоставить замкнутый путь длины $k$ на графе $\mathcal{B}_{2 n}$. С другой стороны, лемма 5 утверждает, что всякому пути длины $k$ можно сопоставить периодическую точку отображения $f$. Другое дело, что период той точки может не быть равен $k$, однако он обязан быть делителем этого числа. Следовательно, имеет место равенство

$$
\ell_{k}=\sum_{d \mid k} p_{d}
$$

из которого, в силу формулы обращения Мёбиуса (см., к примеру, [9, с. 96, теорема 3.19]), следует, что

$$
p_{k}=\sum_{d \mid k} \mu(d) \ell_{k / d} .
$$


Осталось заметить, что число траекторий периода $k$ в $k$ раз меньше числа периодических точек периода $k$, что и завершает доказательство.

6. Заключительные замечания. Рассмотрим частный случай $m=5$. В табл. 1 приведено число траекторий периода $k$ отображения $f:[1 ; 5] \rightarrow[1 ; 5]$ для не очень больших $k$.

Таблица 1.

\begin{tabular}{|c|c|c|c|c|c|c|c|c|c|c|c|c|c|c|c|c|c|c|}
\hline$k$ & 1 & 2 & 3 & 4 & 5 & 6 & 7 & 8 & 9 & 10 & 11 & 12 & 13 & 14 & 15 & 16 & 17 & 18 \\
\hline traj $_{k}$ & 1 & 1 & 0 & 1 & 1 & 2 & 2 & 3 & 4 & 6 & 8 & 11 & 16 & 23 & 32 & 46 & 66 & 94 \\
\hline
\end{tabular}

Конечно, эти числа растут экспоненциально. Обозначим через $\lambda_{1}$ единственный положительный корень многочлена $x^{5}-2 x^{3}-1$. Оказывается, что числа $w_{k}=\lambda_{1}^{k} / k$ являются вполне хорошими приближениями для числа траекторий периода $k$. В третьей строке следующей таблицы приведены округленные до натуральных чисел значения $w_{k}$ :

Таблица 2.

\begin{tabular}{|c|c|c|c|c|c|}
\hline$k$ & 56 & 57 & 58 & 59 & 60 \\
\hline $\operatorname{traj}_{k}$ & 209315493 & 311115248 & 462561544 & 687941016 & 1023420097 \\
\hline$w_{k}$ & 209317250 & 311115494 & 462564127 & 687941293 & 1023423976 \\
\hline
\end{tabular}

Поясним это наблюдение при помощи следующего рассуждения. Очевидно, что при $n \geq 2$ многочлен $x^{2 n+1}-2 x^{2 n-1}-1$ имеет три действительных корня: $\lambda_{1} \in\left(\sqrt{2} ; \frac{1+\sqrt{5}}{2}\right),-1$ и $\lambda_{2} \in(-\sqrt{2} ;-1)$. В работе [4] (в которой этот многочлен появился при оценке топологической энтропии) показано, что все его комплексные корни лежат внутри единичного круга, $\left|\lambda_{i}\right|<1, i=3,4, \ldots, 2 n$. Так как $\ell_{k}=\sum_{i=1}^{2 n} \lambda_{i}^{k}$, то, тем самым, $\ell_{k} \sim \lambda_{1}^{k}$. Осталось заметить, что $\operatorname{traj}_{k} \sim \ell_{k} / k$.

В заключение автор выражает свою искреннюю признательность Б. М. Беккеру и Ю. А. Ильину за полезные обсуждения.

\section{Литература}

1. Шарковский А.Н. Сосуществование циклов непрерывного преобразования прямой в себя // Украинский математический журнал. 1964. № 1. С. 61-71.

2. Du. B.-S. The minimal number of periodic orbits of periods guaranteed in Sharkovskii's theorem // Bull. Austral. Math. Soc. 1985. Vol. 31. P. 89-103.

3. Иванов О. А. Оценка числа периодических траекторий данного периода отображений отрезка, числа Люка и ожерелья // Вестник Санкт-Петербургского университета. Математика. Механика. Астрономия. 2018. Т. 5(63). Вып. 4. С. 606-613. https://doi.org/10.21638/11701/spbu01.2018.406

4. Stefan P. A theorem of Sarkovskii on the existence of periodic orbits of continuous endomorphisms of the real line // Comm. Math. Phys. 1977. Vol. 54. P. 237-248.

5. Elaydi S. N. On a Converse of Sharkovsky's Theorem // The American Mathematical Monthly. 1996. Vol. 103, no. 5. Р. 386-392. 1994.

6. Кострикин А. И. Введение в алгебру. Основы алгебры: Учебник для вузов. М.: Физматлит,

7. Шарковский А.Н., Коляда С. Ф., Сивак А. Г., Федоренко В. В. Динамика одномерных отображений. Киев: Наукова Думка, 1989. 
8. Li T.-Y., Yorke J. A. Period three implies chaos // The American Mathematical Monthly. 1975. Vol. 82, no. 10. P. 985-992.

9. Иванов О. А. Элементарная математика для школьников, студентов и преподавателей. М.: МЦНМО, 2009.

$$
\begin{array}{r}
\text { Статья поступила в редакцию } 28 \text { декабря } 2018 \text { г.; } \\
\text { после доработки } 11 \text { февраля } 2019 \text { г.; } \\
\text { рекомендована в печать } 21 \text { марта } 2019 \text { г. }
\end{array}
$$

Контактная информация:

Иванов Олег Александрович — д-р пед. наук, проф.; о.a.ivanov@spbu.ru

\section{Sharkovskii's ordering and evaluations of the number of periodic trajectories of given period of a self-map of an interval}

\section{O. A. Ivanov}

St. Petersburg State University, Universitetskaya nab., 7-9, St. Petersburg, 199034, Russian Federation

For citation: Ivanov O. A. Sharkovskii's ordering and evaluations of the number of periodic trajectories of given period of a self-map of an interval. Vestnik of Saint Petersburg University. Mathematics. Mechanics. Astronomy, 2019, vol. 6 (64), issue 3, pp. 422-429. https://doi.org/10.21638/11701/spbu01.2019.307 (In Russian)

In 1964, A. N. Sharkovskii published an article in which he introduced a special ordering on the set of positive integers. His ordering has the property that if $p \triangleleft q$ and a continuous mapping of an interval into itself has a point of period $p$, then it has a point of period $q$. Since the least number with respect to this ordering is the number 3 , it follows that if such a mapping has a point of period 3, then it has points of every period. The latter result was rediscovered in 1975 by $\mathrm{Li}$ and Yorke, who published it in their paper "Period three implies chaos". Their work led to global recognition of Sharkovskii's theorem, and since then a great number of papers related to the study of mappings of an interval have appeared. One area of research concerns estimates of the number of periodic trajectories a map satisfying the conditions of Sharkovskyii's theorem must have. In 1985, Bau-Sen Du published a paper in which he gave the exact lower bound for the number of periodic trajectories of a given period. The present article provides a new, significantly shorter and more natural proof of his result.

Keywords: Sharkovsky's ordering, mappings of an interval, a periodical trajectory, paths on directed graphs.

\section{References}

1. Sharkovskii A. N., "Co-existence of cycles of a continuous mapping of a line onto itself", International Journal of Bifurcation and Chaos and Applications in Applied Science and Engineering 5, 1263-1273 (1995).

2. Du B.-S., "The minimal number of periodic orbits of periods guaranteed in Sharkovskii's theorem", Bull. Austral. Math. Soc. 31, 89-103 (1985).

3. Ivanov O.A., "An estimate for the number of periodical trajectories of the given period for mapping of an interval, Lucas numbers, and necklaces", Vestnik St. Petersburg University, Mathematics 51, issue 4, 367-372 (2018). https://doi.org/10.3103/S1063454118040088

4. Štefan P., "A theorem of Šarkovskii on the existence of periodic orbits of continuous endomorphisms of the real line", Comm. Math. Phys. 54, 237-248 (1977).

5. Elaydi S. N., "On a Converse of Sharkovsky's Theorem", The American Mathematical Monthly 103(5), 386-392 (1996). 
6. Kostrikin A. I., Introduction to algebra. Principles of algebra (Fizmatlit Publ., Moscow, 1994). (In Russian)

7. Sharkovsky A. N., Kolyada S.F., Sivak A.G., Fedorenko F.F., Dynamics of One-Dimensional Maps (Kluwer Academic Publishers, 1997).

8. Li T.-Y., Yorke J. A., "Period three implies chaos", The American Mathematical Monthly 82(10), 985-992 (1975).

9. Ivanov O. A., Making Mathematics Come to Life: A Guide for Teachers and Students (American Mathematical Society, Providence, RI, 2009).

Received: December 28, 2018

Revised: February 11, 2019

Accepted: March 21, 2019

Author's information:

Oleg A. Ivanov-o.a.ivanov@spbu.ru 